\title{
Quenching Spin Decoherence in Diamond through Spin Bath Polarization
}

\author{
Susumu Takahashi, ${ }^{1, *}$ Ronald Hanson, ${ }^{2,3}$ Johan van Tol, ${ }^{4}$ Mark S. Sherwin, ${ }^{1}$ and David D. Awschalom ${ }^{3}$ \\ ${ }^{1}$ Department of Physics and Center for Terahertz Science and Technology, University of California, \\ Santa Barbara, California 93106, USA \\ ${ }^{2}$ Kavli Institute of Nanoscience, Delft University of Technology, P.O. Box 5046, 2600 GA Delft, The Netherlands \\ ${ }^{3}$ Department of Physics and Center for Spintronics and Quantum Computation, University of California, \\ Santa Barbara, California 93106, USA \\ ${ }^{4}$ National High Magnetic Field Laboratory, Florida State University, Tallahassee Florida 32310, USA
}

(Received 8 April 2008; published 23 July 2008)

\begin{abstract}
We experimentally demonstrate that the decoherence of a spin by a spin bath can be completely eliminated by fully polarizing the spin bath. We use electron paramagnetic resonance at $240 \mathrm{GHz}$ and $8 \mathrm{~T}$ to study the electron-spin coherence time $T_{2}$ of nitrogen-vacancy centers and nitrogen impurities in diamond from room temperature down to $1.3 \mathrm{~K}$. A sharp increase of $T_{2}$ is observed below the Zeeman energy $(11.5 \mathrm{~K})$. The data are well described by a suppression of the flip-flop induced spin bath fluctuations due to thermal electron-spin polarization. $T_{2}$ saturates at $\sim 250 \mu \mathrm{s}$ below $2 \mathrm{~K}$, where the polarization of the electron-spin bath exceeds $99 \%$.
\end{abstract}

DOI: 10.1103/PhysRevLett.101.047601

PACS numbers: 76.30.Mi, 03.65.Yz

Overcoming spin decoherence is critical to spintronics and spin-based quantum information processing devices $[1,2]$. For spins in the solid state, a coupling to a fluctuating spin bath is a major source of the decoherence. Therefore, several recent theoretical and experimental efforts have aimed at suppressing spin bath fluctuations [3-9]. One approach is to bring the spin bath into a well-known quantum state that exhibits little or no fluctuations $[10,11]$. A prime example is the case of a fully polarized spin bath. The spin bath fluctuations are fully eliminated when all spins are in the ground state. In quantum dots, nuclear spin bath polarizations of up to $60 \%$ have been achieved [12,13]. However, a polarization above $90 \%$ is needed to significantly increase the spin coherence time [14]. Moreover, thermal polarization of the nuclear spin bath is experimentally challenging due to the small nuclear magnetic moment. Electron-spin baths, however, may be fully polarized thermally at a few degrees of Kelvin under an applied magnetic field of $8 \mathrm{~T}$.

Here we investigate the relationship between the spin coherence of nitrogen-vacancy $(\mathrm{N}-\mathrm{V})$ centers in diamond and the polarization of the surrounding spin bath consisting of nitrogen $(\mathrm{N})$ electron spins. N-V centers consist of a substitutional nitrogen atom adjoining to a vacancy in the diamond lattice. The N-V center, which has long spin coherence times at room temperature $[15,16]$, is an excellent candidate for quantum information processing applications as well as conducting fundamental studies of interactions with nearby electronic spins [16-18] and nuclear spins $[19,20]$. In the case of type-Ib diamond, as studied here, the coupling to a bath of $\mathrm{N}$ electron spins is the main source of decoherence for an $\mathrm{N}-\mathrm{V}$ center spin $[15,21]$. We have measured the spin coherence time $\left(T_{2}\right)$ and spin-lattice relaxation time $\left(T_{1}\right)$ in spin ensembles of $\mathrm{N}-\mathrm{V}$ centers and single $\mathrm{N}$ impurity centers ( $P 1$ centers) using pulsed electron paramagnetic resonance (EPR) spec- troscopy at $240 \mathrm{GHz}$. By comparing the values of $T_{1}$ and $T_{2}$ at different temperatures, we verify that the mechanism determining $T_{2}$ is different from that of $T_{1}$. Next, we investigate the temperature dependence of $T_{2}$.

At $240 \mathrm{GHz}$ and $8.6 \mathrm{~T}$ where the Zeeman energy of the $\mathrm{N}$ centers corresponds to $11.5 \mathrm{~K}$, the polarization of the $\mathrm{N}$ spin bath is almost complete $(99.4 \%)$ for temperatures below $2 \mathrm{~K}$ as shown in Fig. 1(a). This extremely high polarization has a dramatic effect on the spin bath fluctuations, and thereby on the coherence of the $\mathrm{N}-\mathrm{V}$ center spin. We find that $T_{2}$ of the N-V center spin is nearly constant between room temperature and $20 \mathrm{~K}$, but increases by almost 2 orders of magnitude below the Zeeman energy to a saturation value of $\sim 250 \mu \mathrm{s}$ at $2 \mathrm{~K}$. The data show excellent agreement with a model based on spin flip-flop processes in the spin bath. The observed saturation value suggests that when the $\mathrm{N}$ spin bath is fully polarized, $T_{2}$ is limited by the fluctuations in the ${ }^{13} \mathrm{C}$ nuclear spin bath.

We studied a single crystal of high-temperature highpressure type-Ib diamond, which is commercially available from Sumitomo electric industries. The density of $\mathrm{N} \mathrm{im-}$ purities is $10^{19}$ to $10^{20} \mathrm{~cm}^{-3}$. The sample was irradiated with $1.7 \mathrm{MeV}$ electrons with a dose of $5 \times 10^{17} \mathrm{~cm}^{-3}$ and subsequently annealed at $900{ }^{\circ} \mathrm{C}$ for $2 \mathrm{~h}$ to increase the $\mathrm{N}-\mathrm{V}$ concentration [22].

Electronic spin Hamiltonians for the $\mathrm{N}-\mathrm{V}\left(H_{\mathrm{NV}}\right)$ and $\mathrm{N}$ centers $\left(H_{\mathrm{N}}\right)$ are,

$$
\begin{gathered}
H_{\mathrm{NV}}=D\left[\left(S_{z}^{\mathrm{NV}}\right)^{2}-\frac{1}{3} S^{\mathrm{NV}}\left(S^{\mathrm{NV}}+1\right)\right]+\mu_{B} g^{\mathrm{NV}} \boldsymbol{S}^{\mathrm{NV}} \cdot \boldsymbol{B}_{0} \\
+A^{\mathrm{NV}} S^{\mathrm{NV}} \cdot \boldsymbol{I}^{\mathrm{N}}, \\
H_{\mathrm{N}}=\mu_{B} \boldsymbol{S}^{\mathrm{N}} \cdot \stackrel{\leftrightarrow}{g^{\mathrm{N}}} \cdot \boldsymbol{B}_{0}+A^{\mathrm{N}} \boldsymbol{S}^{\mathrm{N}} \cdot \boldsymbol{I}^{\mathrm{N}},
\end{gathered}
$$

where $\mu_{B}$ is the Bohr magneton and $\boldsymbol{B}_{0}$ is the magnetic field. $S^{\mathrm{NV}}$ and $\boldsymbol{S}^{\mathrm{N}}$ are the electronic spin operators for the $\mathrm{N}-\mathrm{V}$ and $\mathrm{N}$ centers and $\boldsymbol{I}^{\mathrm{N}}$ is the nuclear spin operator for 

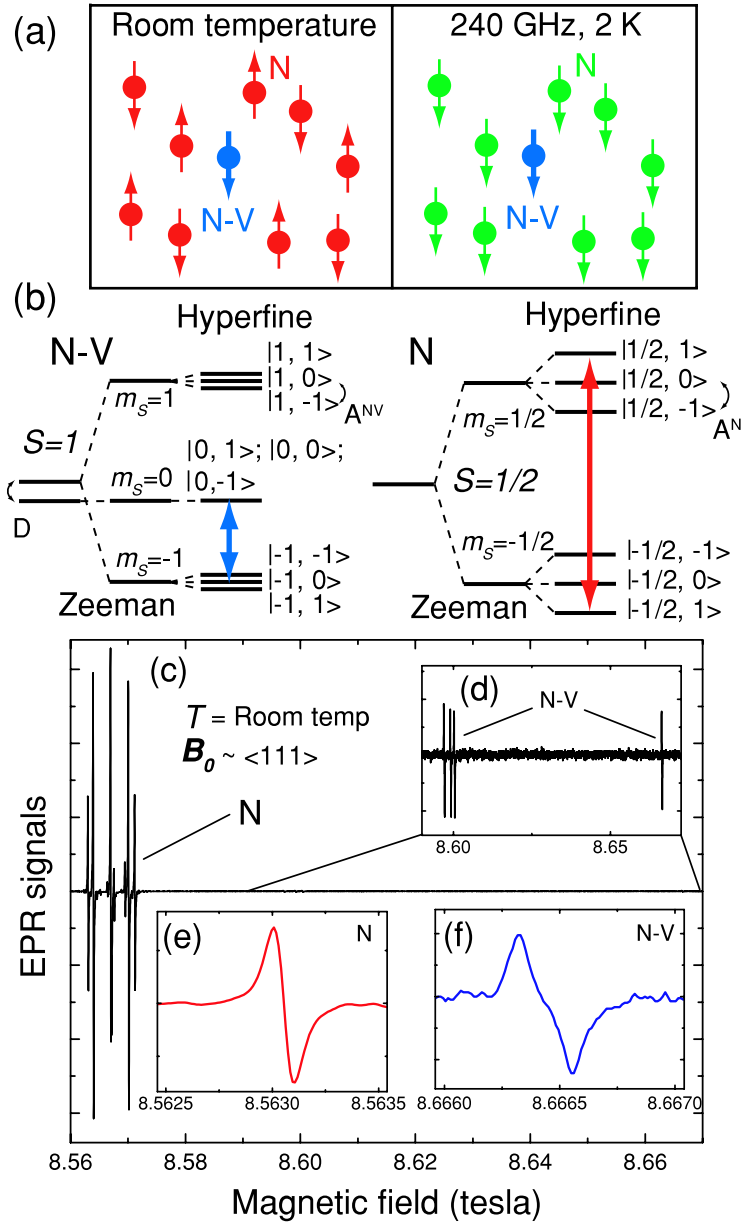

FIG. 1 (color online). (a) Spins of the N-V and N centers at room temperature and at $8.56 \mathrm{~T}$ and $2 \mathrm{~K}$. At room temperature, where up and down spins are nearly equally populated, the $\mathrm{N}$ spin bath polarization is very small and therefore, the spin flipflop rate is high. At $240 \mathrm{GHz}$ and $2 \mathrm{~K}$, the $\mathrm{N}$ spin bath polarization is $99.4 \%$ and the spin flip-flop rate is nearly zero. (b) Energy states of the N-V and N centers. The energy levels are not scaled. The states are indexed by $\left|m_{S}, m_{I}\right\rangle$. Solid lines indicate EPR transitions used to measure the spin relaxation times $T_{1}$ and $T_{2}$. (c) cw EPR spectrum at $240 \mathrm{GHz}$ at room temperature. No optical pump is applied. The strongest five EPR peaks around $8.57 \mathrm{~T}$ are from $\mathrm{N}$ centers. (d) N-V EPR peaks. The intensity ratio between the left-most $\mathrm{N}$ and the right-most $\mathrm{N}-\mathrm{V}$ is $\sim 80$ which corresponds to $120: 1$ population ratio between $\mathrm{N}$ and $\mathrm{N}-\mathrm{V}$ centers, respectively. Other impurity centers were also observed (not indicated). (e) N centers EPR for the transition of $\left|m_{S}=-1 / 2, m_{I}=1\right\rangle \leftrightarrow|1 / 2,1\rangle$. (f) $\mathrm{N}-\mathrm{V}$ centers EPR for the transition of $\left|m_{S}=-1\right\rangle \leftrightarrow|0\rangle$.

${ }^{14} \mathrm{~N}$ nuclear spins. $g^{\mathrm{NV}}=2.0028$ [23], and $\stackrel{\leftrightarrow}{g^{\mathrm{N}}}$ is the slightly anisotropic $g$-tensor of the $\mathrm{N}$ center. $D=$ $2.87 \mathrm{GHz}$ is the zero-field splitting due to the axial crystal field [23]. Because of the tetrahedral symmetry of diamond lattice, there are four possible orientations of the defect principal axis of the ${ }^{14} \mathrm{~N}$ hyperfine coupling of $A^{\mathrm{N}}$ and $A^{\mathrm{NV}}$. In the present case, $A^{\mathrm{N}}=114 \mathrm{MHz}$ for the $\langle 111\rangle$-orientation and $A^{\mathrm{N}}=86 \mathrm{MHz}$ for the other three orientations [24]. For the N-V center, $A^{\mathrm{NV}}=2.2 \mathrm{MHz}$ for the $\langle 111\rangle$-orientation [23]. The hyperfine coupling between the $\mathrm{N}-\mathrm{V}(\mathrm{N})$ center and ${ }^{13} \mathrm{C}$ and the nuclear Zeeman energy are not included here. The energy states of the $\mathrm{N}-\mathrm{V}$ and $\mathrm{N}$ centers are shown in Fig. 1(b).

The measurement was performed using a $240 \mathrm{GHz}$ continuous wave (cw) and pulsed EPR spectrometer in the electron magnetic resonance program at the National High Magnetic Field Laboratory (NHMFL), Tallahassee FL. The setup is based on a superheterodyne quasioptical bridge with a $40 \mathrm{~mW}$ solid state source. Details of the EPR setup are described elsewhere [25,26]. No optical excitation was applied throughout this work, and no resonator was used for either $\mathrm{cw}$ or pulsed experiments. Figures 1(c)-1(f) show cw EPR spectra at room temperature where the magnetic field was applied along the $\langle 111\rangle$-direction of the $\sim 0.8 \times 0.8 \times 0.6 \mathrm{~mm}^{3}$ single crystal diamond. The applied microwave power and field modulation intensity were carefully tuned not to distort the EPR line shape. Five EPR spectra in Fig. 1(c) corresponding to the $\mathrm{N}$ center are drastically stronger than the remaining signals which indicates that the number of $\mathrm{N}$ centers dominates the spin population in the sample. The $\mathrm{N}$ EPR peaks show the slightly anisotropic $g$ factor $g^{\mathrm{N}}$ which gives $g_{\|}^{\mathrm{N}}=2.0024$ and $g_{\perp}^{\mathrm{N}}=2.0025-6$ and is in agreement with the reported $g$-anisotropy of type-IIa diamond [27]. As shown in Fig. 1(d), we also observed the much smaller $\mathrm{N}-\mathrm{V}$ resonances which shows a line for the $\langle 111\rangle$-orientation at higher magnetic field and three lines for the other orientations at lower magnetic field. An overlap of the three lines is lifted because the applied $B_{0}$ field is slightly tilted from the $\langle 111\rangle$-direction. Based on the EPR intensity ratio between $\mathrm{N}$ and $\mathrm{N}-\mathrm{V}$ centers, the estimated density of the $\mathrm{N}-\mathrm{V}$ centers in the studied sample is approximately $10^{17}$ to $10^{18} \mathrm{~cm}^{-3}$. EPR line shapes of the $\mathrm{N}\left(\left|m_{S}=-1 / 2, m_{I}=1\right\rangle \leftrightarrow|1 / 2,1\rangle\right)$ and $\mathrm{N}-\mathrm{V}$ $\left(\left|m_{S}=-1\right\rangle \leftrightarrow|0\rangle\right)$ centers are shown in Fig. 1(e) and 1(f) respectively. The $\mathrm{N}$ center shows a single EPR line with a peak-to-peak width of $0.95 \mathrm{G}$. On the other hand, the $\mathrm{N}-\mathrm{V}$ center shows a broader EPR line (the peak-to-peak width is $2.36 \mathrm{G}$ ) due to the hyperfine coupling between the $\mathrm{N}-\mathrm{V}$ center and the ${ }^{14} \mathrm{~N}$ nuclear spins. The estimated hyperfine constant is $2 \mathrm{MHz}$, in good agreement with previous findings [23].

The temperature dependence of the spin relaxation times $T_{1}$ and $T_{2}$ was measured using pulsed EPR. An echodetected inversion recovery sequence $(\pi-T-\pi / 2-$ $\tau-\pi-\tau-$ echo) is applied for $T_{1}$ where a delay $T$ is varied, while a Hahn echo sequence $(\pi / 2-\tau-\pi-\tau-$ echo) is applied for $T_{2}$ where a delay $\tau$ is varied [28]. The area of the echo signal decays as a function of the delay time $T$ and $2 \tau$ for $T_{1}$ and $T_{2}$, respectively, and therefore can be used to determine the relaxation times. For the pulsed EPR measurement, we used the $\left|m_{S}=-1, m_{I}=0\right\rangle \leftrightarrow$ 


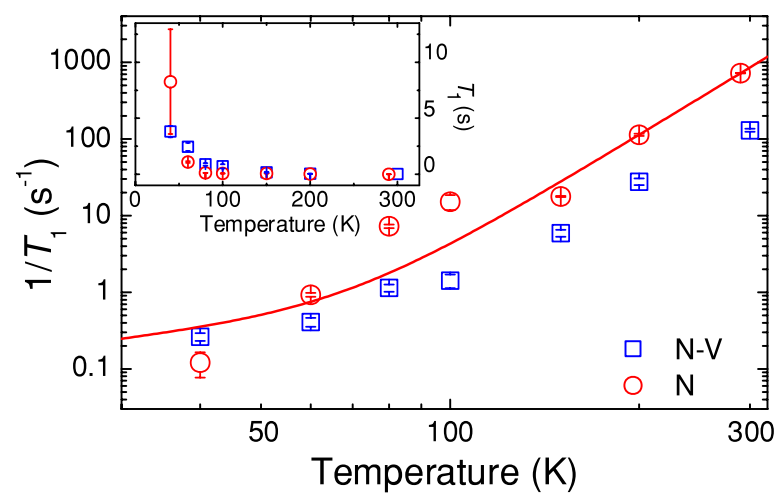

FIG. 2 (color online). $\quad 1 / T_{1}$ for the $\mathrm{N}-\mathrm{V}$ and $\mathrm{N}$ centers as a function of temperature. Solid lines are the best fit of the spinorbit phonon-induced tunneling model. Inset of the graph shows $T_{1}$ versus temperature in a linear scale.

$|0,0\rangle$ transition for the $\mathrm{N}-\mathrm{V}$ center and the $\left|m_{S}=-1 / 2, m_{I}=1\right\rangle \leftrightarrow|1 / 2,1\rangle$ transition for the $\mathrm{N}$ center [Fig. 1(b)].

$T_{1}$ for both the N-V and $\mathrm{N}$ centers was measured from room temperature to $40 \mathrm{~K}$. Below $40 \mathrm{~K}$, where $T_{1}$ is longer than tens of seconds $[29,30]$, an accurate measurement with the current setup proved impractical as the drift of the superconducting magnet $(\sim 5 \mathrm{ppm} / \mathrm{h})$ becomes nontrivial on the timescale of the measurement. $T_{1}$ is obtained by fitting a decay exponential to the recovery rate of the echo area $y_{0}-a e^{-T / T_{1}}$. As shown in the inset of Fig. 2, $T_{1}$ of both centers increases significantly as the temperature is reduced. For the N-V center, $T_{1}$ changes from $7.7 \pm$ $0.4 \mathrm{~ms}$ to $3.8 \pm 0.5 \mathrm{~s}$. For the $\mathrm{N}$ center, $T_{1}$ increases from $1.38 \pm 0.01 \mathrm{~ms}$ to $8.3 \pm 4.7 \mathrm{~s}$. For the spin-lattice relaxation of the $\mathrm{N}$ centers, it was found that a spin-orbit induced tunneling model is the main mechanism [29]. We fit our data to their model given by $1 / T_{1}=A T+B T^{5}$, where $A$ and $B$ are parameters. The obtained fit parameters $A=8.0 \times 10^{-3}$ and $B=3.5 \times 10^{-10}$ are close to the values found in Ref. [29]. It was, however, noticed that although the fit explains the main trend, there is a significant deviation for several data points. For the $\mathrm{N}-\mathrm{V}$ center, a previous study hinted at a two-phonon Orbach process as the main relaxation mechanism [31]. However, the temperature dependence observed here does not fit well to this model. These deviations may be related to effects of the $\mathrm{N}$ spin bath and spectral diffusion in the $\mathrm{N}$ and $\mathrm{N}-\mathrm{V}$ spins. A detailed investigation is beyond the scope of the present work and will be presented elsewhere.

We also investigated the temperature dependence of the spin coherence time $T_{2}$ for the N-V center using a Hahn echo sequence where the width of the pulses (typically 500-700 ns) was tuned to maximize the echo size. Figure 3(a) shows the decay of echo area at room temperature and at $T=1.3 \pm 0.1 \mathrm{~K}$. These decays, which are well fit by a single exponential $e^{-2 \tau / T_{2}}$ as shown in Fig. 3(a), show no evidence of electron-spin echo envelope modula-

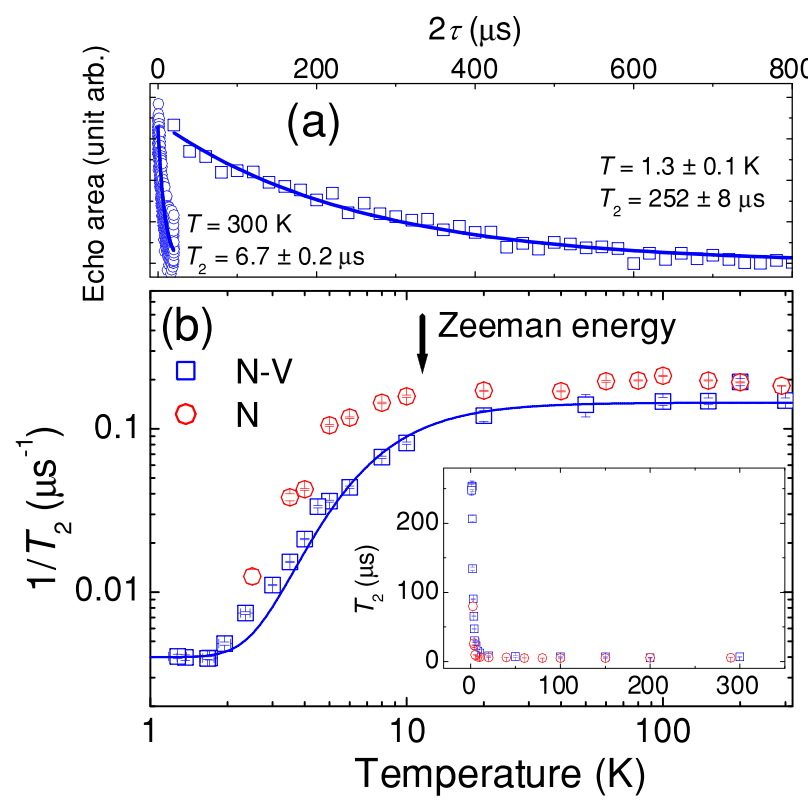

FIG. 3 (color online). (a) Echo area of the N-V center as a function of delay $2 \tau$ measured at room temperature and $T=$ $1.3 \pm 0.1 \mathrm{~K}$. Solid line shows the best fit by the single exponential. (b) $1 / T_{2}$ for the N-V and $\mathrm{N}$ centers versus temperature. The scale of the main graph is $\log$-log. Solid lines are the best fit using Eq. (3). The arrow shows the Zeeman energy of $11.5 \mathrm{~K}$. The inset shows $T_{2}$ versus temperature in linear scale which shows a dramatic increase of $T_{2}$ below the Zeeman energy.

tion (ESEEM) effects from the ${ }^{14} \mathrm{~N}$ hyperfine coupling [28]. This is due to the relative long microwave pulses and the nuclear Zeeman splitting at $8.5 \mathrm{~T}$ which is much larger than the ${ }^{14} \mathrm{~N}$ hyperfine coupling of the $\mathrm{N}-\mathrm{V}$ center. Between room temperature and $20 \mathrm{~K}$, we observe almost no temperature dependence with $T_{2} \ll T_{1}$, (e.g., $T_{2}=$ $6.7 \pm 0.2 \mu \mathrm{s}$ at room temperature and $T_{2}=8.3 \pm$ $0.7 \mu \mathrm{s}$ at $20 \mathrm{~K}$ ). This verifies that the mechanism which determines $T_{2}$ is different from that of $T_{1}$. Below the Zeeman energy (11.5 K), $T_{2}$ increases drastically as shown in the inset of Fig. 3(b). By lowering the temperature further, $T_{2}$ increases up to $\sim 250 \mu \mathrm{s}$ at $1.7 \mathrm{~K}$ and does not show noticeable increase below $1.7 \mathrm{~K}$.

At high magnetic field, where single spin flips are suppressed, the fluctuations in the bath are mainly caused by energy-conserving flip-flop processes of the $\mathrm{N}$ spins. The spin flip-flop rate in the bath is proportional to the number of pairs with opposite spin and thus it strongly depends on the spin bath polarization [32]. At $240 \mathrm{GHz}$ and $2 \mathrm{~K}$, the $\mathrm{N}$ spin bath polarization is $99.4 \%$ which almost eliminates the spin flip-flop process. This experiment therefore verifies that the dominant decoherence mechanism of the $\mathrm{N}-\mathrm{V}$ center in type-Ib diamond is the spin-flop process of the $\mathrm{N}$ spin bath. Using the partition function for the Zeeman term of the $\mathrm{N}$ spins, $Z=\sum_{S=-1 / 2}^{1 / 2} e^{-\beta \mu_{B} g^{N} B_{0} S}$, where $\beta=$ $1 /\left(k_{B} T\right)$ and $k_{B}$ is Boltzmann constant, the flip-flop rate is modeled by the following equation [32], 


$$
\begin{aligned}
\frac{1}{T_{2}} & \equiv C P_{m_{S}=-1 / 2} P_{m_{S}=1 / 2}+\Gamma_{\text {res }} \\
& =\frac{C}{\left(1+e^{T_{\mathrm{ze}} / T}\right)\left(1+e^{-T_{\mathrm{ze}} / T}\right)}+\Gamma_{\mathrm{res}},
\end{aligned}
$$

where $C$ is a temperature independent parameter, $T_{\mathrm{Ze}}$ is the temperature corresponding to Zeeman energy and $\Gamma_{\text {res }}$ is a residual relaxation rate. We fit the $T_{2}$ data for the N-V center using the equation above. The fit was performed with the fixed $\Gamma_{\text {res }}=0.004\left(\mu \mathrm{s}^{-1}\right)$ corresponding to $250 \mu \mathrm{s}$. This model fit the data well as shown in the log scale plot of Fig. 3(b). $T_{\mathrm{Ze}}=14.7 \pm 0.4 \mathrm{~K}$ obtained from the fit is in reasonable agreement with the actual Zeeman energy of $11.5 \mathrm{~K}$. The result thus confirms the decoherence mechanism of the $\mathrm{N}$ spin bath fluctuation.

The observation of the saturation of $T_{2} \sim 250 \mu \mathrm{s}$ also indicates complete quenching of the $\mathrm{N}$ spin bath fluctuation and a second decoherence source in this system. From previous studies $[16,19]$, the most probable second source is a coupling to the ${ }^{13} \mathrm{C}$ nuclear spin bath. In fact, $T_{2} \sim$ $250 \mu$ s agrees with an estimated decoherence time of ${ }^{13} \mathrm{C}$ spin bath fluctuations [16].

Finally, we investigate temperature dependence of $T_{2}$ for the $\mathrm{N}$ center at $240 \mathrm{GHz}$. No temperature dependence of $T_{2}$ was observed in a previous pulsed EPR study at $9.6 \mathrm{GHz}$ [29]. We measured the $\left|m_{S}=-1 / 2, m_{I}=1\right\rangle \leftrightarrow|1 / 2,1\rangle$ transition shown in Fig. 1(b) which can excite only 1/12 of the $\mathrm{N}$ center population while it is assumed that all $\mathrm{N}$ spins in this transition are on resonance [24]. The temperature dependence of $T_{2}$ therefore shows the relationship between $1 / 12$ of the $N$ center and $11 / 12$ of the $N$ spin bath fluctuation. Similar to the N-V center, we found slight change between room temperature and $20 \mathrm{~K}$, i.e., $T_{2}=5.455 \pm$ $0.005 \mu \mathrm{s}$ at room temperature and $T_{2}=5.83 \pm 0.04 \mu \mathrm{s}$ at $20 \mathrm{~K}$, and then a significant increase below the Zeeman energy. Eventually, $T_{2}$ becomes $80 \pm 9 \mu$ s at $2.5 \mathrm{~K}$. As shown in Fig. 3(b), the temperature dependence of $T_{2}$ is similar to that of the $\mathrm{N}-\mathrm{V}$ center. These facts support strongly that the decoherence mechanism of the $\mathrm{N}$ center is also the $\mathrm{N}$ spin bath fluctuation.

In conclusion, we presented the temperature dependence of the spin relaxation times $T_{1}$ and $T_{2}$ of the N-V and $\mathrm{N}$ centers in diamond. The temperature dependence of $T_{2}$ confirms that the primary decoherence mechanism in type-Ib diamond is the $\mathrm{N}$ spin bath fluctuation. We have demonstrated that we can strongly polarize the $\mathrm{N}$ spin bath and quench its decoherence. We observed that $T_{2}$ of the $\mathrm{N}-\mathrm{V}$ center saturates $\sim 250 \mu$ s below $2 \mathrm{~K}$ which indicates a secondary decoherence mechanism and is in good agreement with an estimated coherence time dominated by ${ }^{13} \mathrm{C}$ nuclear spin fluctuations. These measurements demonstrate that high-frequency pulsed EPR at low temperatures provides access to a new regime of spin decoherence in solids.

This work was supported by research grants from NSF (DMR-0520481) and the W. M. Keck Foundation (M. S. S. and S. T.), FOM and NWO (R. H.) and AFOSR (D. D. A.). S. T. thanks the NHMFL EMR program for travel support.

*susumu@iqcd.ucsb.edu

[1] D. D. Awschalom and M. E. Flatté, Nature Phys. 3, 153 (2007).

[2] R. Hanson et al., Rev. Mod. Phys. 79, 1217 (2007).

[3] A. V. Khaetskii, D. Loss, and L. Glazman, Phys. Rev. Lett. 88, 186802 (2002).

[4] I. A. Merkulov, A. L. Efros, and M. Rosen, Phys. Rev. B 65, 205309 (2002).

[5] R. de Sousa and S. DasSarma, Phys. Rev. B 68, 115322 (2003).

[6] V. V. Dobrovitski, H. A. DeRaedt, M. I. Katsnelson, and B. N. Harmon, Phys. Rev. Lett. 90, 210401 (2003).

[7] F. M. Cucchietti, J. P. Paz, and W. H. Zurek, Phys. Rev. A 72, 052113 (2005).

[8] J. M. Taylor and M.D. Lukin, Quant. Info. Proc. 5, 503 (2006).

[9] W. Yao, R.-B. Liu, and L. J. Sham, Phys. Rev. Lett. 98, 077602 (2007).

[10] D. Stepanenko, G. Burkard, G. Giedke, and A. Imamoglu, Phys. Rev. Lett. 96, 136401 (2006).

[11] D. Klauser, W. A. Coish, and D. Loss, Phys. Rev. B 73, 205302 (2006).

[12] A. S. Bracker et al., Phys. Rev. Lett. 94, 047402 (2005).

[13] J. Baugh, Y. Kitamura, K. Ono, and S. Tarucha, Phys. Rev. Lett. 99, 096804 (2007).

[14] W. A. Coish and D. Loss, Phys. Rev. B 70, 195340 (2004).

[15] T. A. Kennedy et al., Appl. Phys. Lett. 83, 4190 (2003).

[16] T. Gaebel et al., Nature Phys. 2, 408 (2006).

[17] R. Hanson, F. M. Mendoza, R. J. Epstein, and D. D. Awschalom, Phys. Rev. Lett. 97, 087601 (2006).

[18] R. Hanson et al., Science 320, 352 (2008).

[19] L. Childress et al., Science 314, 281 (2006).

[20] M. V. G. Dutt et al., Science 316, 1312 (2007).

[21] R. Hanson, O. Gywat, and D. D. Awschalom, Phys. Rev. B 74, 161203(R) (2006).

[22] R. J. Epstein, F. M. Mendoza, Y. K. Kato, and D. D. Awschalom, Nature Phys. 1, 94 (2005).

[23] J. H. N. Loubser and J. A. vanWyk, Rep. Prog. Phys. 41, 1201 (1978).

[24] W. V. Smith, P. P. Sorokin, I. L. Gelles, and G. J. Lasher, Phys. Rev. 115, 1546 (1959).

[25] J. van Tol, L.-C. Brunel, and R. J. Wylde, Rev. Sci. Instrum. 76, 074101 (2005).

[26] G. W. Morley, L.-C. Brunel, and J. van Tol, Rev. Sci. Instrum. 79, 064703 (2008).

[27] S. Zhang et al., Phys. Rev. B 49, 15392 (1994).

[28] A. Schweiger and G. Jeschke, in Principles of Pulse Electron Paramagnetic Resonance (Oxford University Press, New York, 2001).

[29] E. C. Reynhardt, G. L. High, and J. A. vanWyk, J. Chem. Phys. 109, 8471 (1998).

[30] J. Harrison, M. J. Sellars, and N. B. Manson, Diam. Relat. Mater. 15, 586 (2006).

[31] D. A. Redman, S. Brown, R. H. Sands, and S.C. Rand, Phys. Rev. Lett. 67, 3420 (1991).

[32] C. Kutter et al., Phys. Rev. Lett. 74, 2925 (1995). 